\title{
Persistent Transition Region Emission in Very Low Mass Stars
}

\author{
Suzanne L. Hawley \\ University of Washington, Department of Astronomy, Box 351580, \\ Seattle, WA 98195, USA \\ Christopher M. Johns-Krull \\ Department of Physics \& Astronomy, 6100 Main St., Rice University, \\ Houston, Texas, 77005, USA
}

\begin{abstract}
.
We have obtained HST/STIS time series observations of active, very low mass stars of spectral types M7-M9. The observations comprise 15 consecutive exposures on each of the stars VB 8, VB 10, and LHS 2065. Emission is present in several transition region lines including Si IV, C IV and He II. The emission appears to be variable between exposures, but is always observed. A strong flare was observed in one exposure on VB 10. Our results suggest that active, very low-mass stars maintain a persistent quiescent chromosphere and transition region that is similar to those observed in active, earlier type $\mathrm{M}$ dwarfs. This contradicts the currently popular idea that very low mass stars exhibit only relatively strong flares and no quiescent emission.
\end{abstract}

\section{Introduction}

Chromospheric observations of $\mathrm{H} \alpha$ emission (Hawley et al. 1996; Gizis et al. 2000; Burgasser et al. 2002) indicate that both the frequency of active stars at a given spectral type, and the activity level (measured by $\mathrm{L}_{H \alpha} / L_{b o l}$ ) decrease at spectral types later than approximately M7. Recent investigations of magnetic activity in these very late type dwarfs have led to a new, yet already widely accepted, paradigm: that hot gas in the atmospheres of very low mass main sequence stars and brown dwarfs is present only during flares (Fleming et al. 2000; Rutledge et al. 2000; Mohanty et al. 2002). Here, hot gas refers to gas at transition region and coronal temperatures. The M9 brown dwarf, LP94420, observed with Chandra by Rutledge et al. (2000) does show persistent chromospheric $(\mathrm{H} \alpha)$ emission during quiescence (i.e. outside of flares), as do many of its counterparts.

This new paradigm is based on the following evidence. Linsky et al. (1995) observed the M8 dwarf VB 10 with HST/GHRS and found no quiescent transition region emission, but did observe C IV emission during a flare. Fleming et al. (2000) re-analyzed ROSAT data on VB10 and found that the observed $\mathrm{X}$-ray emission came only from a flare event, and no X-rays were detected during 
six hours of quiescent observation. The X-ray flare luminosity was substantially larger than expected based on the upper limit of the quiescent X-ray luminosity, when compared to flares on earlier type (M0 - M6) stars. An $\mathrm{H} \alpha$ flare on an M9.5 2MASS dwarf showed a similarly substantial flare luminosity compared to quiescence (Liebert et al. 1999). Finally, the afore-mentioned Chandra investigation of the M9 brown dwarf LP944-20 detected no X-rays in a quiescent observation of nine hour duration (Rutledge et al. 2000). On the theoretical side, both Fleming et al. (2000) and Mohanty et al. (2002) have proposed mechanisms related to the low ionization state of very cool atmospheres which would prevent the occurrence of magnetic activity in very cool (hence late spectral type) dwarfs, in order to explain the various observations described above.

Extensive observations of earlier M dwarfs (type M0-M6) have led to scaling relations between the persistent $\mathrm{H} \alpha$ (chromospheric) and soft X-ray (thermal coronal) emission outside of flares (Hawley et al. 1996). We have used $\mathrm{H} \alpha$ observations (Hawley et al. 1996) and C IV (transition region) measurements (Johns-Krull et al. 2000) for the dMe stars AD Leo (M3), EV Lac (M3.5), and YZ CMi (M4) to derive empirical C IV to $\mathrm{H} \alpha$ line flux ratios of $0.06,0.07$, and 0.13 respectively. There may be a tendency for this ratio to increase toward later spectral types. A conservative estimate suggests that the ratio is typically $\sim 0.1$. In terms of the observed fluxes, the relations between chromospheric, transition region, and coronal diagnostics for active early-mid $\mathrm{M}$ dwarfs are therefore:

$$
\begin{aligned}
F_{C I V} & \sim 0.1 F_{H \alpha} \\
F_{X} & \sim 3 F_{H \alpha}
\end{aligned}
$$

If these relations are applied to the observed $\mathrm{H} \alpha$ fluxes for VB 10 and LP94420, the upper limits for the reported non-detections in C IV and X-rays are near or even above the predicted values. For example, Linsky et al. (1995) found an upper limit for VB 10 of $F_{C I V}<6 \times 10^{-16}$ ergs $\mathrm{cm}^{-2} \mathrm{~s}^{-1}$ while the scaling relation gives a predicted C IV line flux of $9 \times 10^{-16} \mathrm{ergs} \mathrm{cm}^{-2} \mathrm{~s}^{-1}$ based on the observed $\mathrm{H} \alpha$ line flux from Hawley et al. (1996). Recently, Martín et al. (2002) reobserved LP944-20 with XMM-Newton, again failing to detect quiescent emission; however, this observation is only a factor of 3 more sensitive than the previous Chandra result of Rutledge et al. (2000). Thus, only slight deviations from the above relations would explain the current non-detections, without requiring a major paradigm shift with regard to magnetic activity on late $\mathrm{M}$ dwarfs compared to earlier $\mathrm{M}$ dwarfs.

\section{Results}

Our target list comprises the brightest active stars known at spectral types M7 (VB 8), M8 (VB 10) and M9 (LHS 2065). These targets were chosen to give us the best possible detection limits for persistent quiescent emission. The total exposure time on each object corresponds to a C IV detection limit ten times fainter than the expected C IV line strength scaled from earlier type active $M$ dwarfs (equation 1).

The Hubble Space Telescope was used with the STIS spectrograph and the FUV-MAMA detector to obtain 15 consecutive exposures of each target. All 
observations were made using the $52^{\prime \prime}$ long by $0.2^{\prime \prime}$ wide slit and the G140L grating to give a spectral resolution $R \equiv \lambda / \delta \lambda \sim 3600$ at $1500 \AA$. For VB 8 , spectral observations started at 12:38 UT on 2001 October 6. Each G140L spectrum was integrated for approximately 5 minutes, and the total exposure sequence spanned two orbits. For LHS 2065, spectral observations started at 5:23 UT on 2002 April 27. Each G140L spectrum was integrated for approximately 10 minutes, and the total exposure sequence spanned four orbits. The observing sequence for VB 10 was identical to that of LHS 2065 with G140L spectral observations starting at 5:03 UT on 2002 September 7 .

Figure 1 shows the time series of observations in the C IV emission lines (which are not resolved at this resolution) obtained for VB 10. Note the large flare in exposure 7, and a possible smaller flare in exposure 9. The emission strength obviously varies significantly between exposures, but is always present.

The measured C IV fluxes for the 15 exposures for each star are given in Table 1 in units of $10^{-15} \mathrm{erg} \mathrm{cm}^{-2} \mathrm{~s}^{-1}$. Values in parentheses next to the C IV flux give the $1 \sigma$ uncertainty in the last two digits of the measured flux.

Table 1. Measured C IV Line Fluxes

\begin{tabular}{cccc}
\hline Exposure & VB 8 & VB 10 & LHS 2065 \\
\hline & & & \\
1 & $2.52(62)$ & $0.69(21)$ & $0.68(21)$ \\
2 & $1.13(46)$ & $1.91(34)$ & $0.58(20)$ \\
3 & $0.91(41)$ & $0.56(21)$ & $0.89(23)$ \\
4 & $1.33(45)$ & $0.18(15)$ & $0.78(24)$ \\
5 & $1.95(54)$ & $1.25(29)$ & $1.15(29)$ \\
6 & $2.43(60)$ & $0.93(26)$ & $1.14(29)$ \\
7 & $2.02(56)$ & $19.8(11)$ & $0.68(20)$ \\
8 & $0.59(37)$ & $2.02(38)$ & $0.75(24)$ \\
9 & $1.04(44)$ & $3.55(49)$ & $0.91(27)$ \\
10 & $0.98(41)$ & $1.35(32)$ & $0.24(16)$ \\
11 & $1.75(52)$ & $1.12(27)$ & $0.34(17)$ \\
12 & $0.52(34)$ & $2.10(39)$ & $0.85(27)$ \\
13 & $2.36(60)$ & $1.84(37)$ & $0.47(21)$ \\
14 & $0.60(34)$ & $2.13(39)$ & $0.67(24)$ \\
15 & $0.52(31)$ & $0.85(24)$ & $0.75(24)$ \\
& & & \\
\hline \hline
\end{tabular}

The C IV line fluxes measured from the integrated spectra are: $1.4 \pm 0.1 \times$ $10^{-15} \mathrm{ergs} \mathrm{s}^{-1} \mathrm{~cm}^{-2}$ for VB $8 ; 2.8(1.4) \pm 0.1(0.1) \times 10^{-15} \mathrm{ergs} \mathrm{s}^{-1} \mathrm{~cm}^{-2}$ for VB 10 ; and $7.1 \pm 0.6 \times 10^{-16} \mathrm{ergs} \mathrm{s}^{-1} \mathrm{~cm}^{-2}$ for LHS 2065 . The values in parentheses for VB 10 represent the quiescent flux and uncertainty measured from the total of all spectra excluding the strong flare observed in exposure 7 (see Figure 1).

The scaling relation based on the $\mathrm{H} \alpha$ emission in these stars, which assumes that the $\mathrm{C}$ IV to $\mathrm{H} \alpha$ ratio is the same as for earlier type $\mathrm{M}$ dwarfs (equation 1), predicted values of $2 \times 10^{-15}$ (VB 8), $9 \times 10^{-16}$ (VB 10), and $1 \times 10^{-15}$ (LHS $2065)$ ergs $\mathrm{s}^{-1} \mathrm{~cm}^{-2}$, i.e. close to the observed values. 


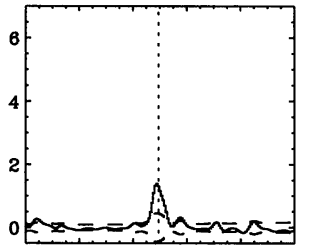

150015201540158015801600
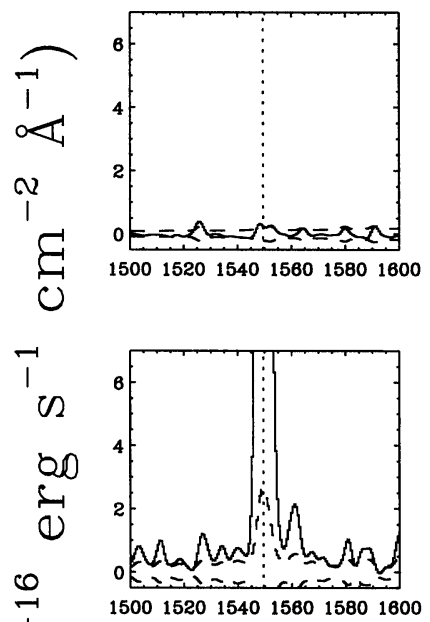

I
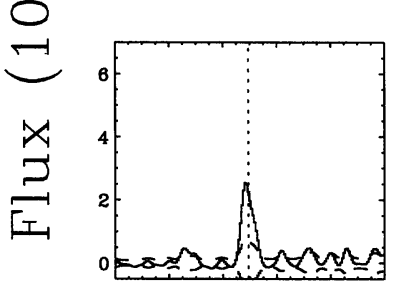

150015201540156015801600

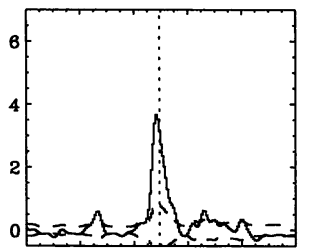

150015201540156015801600
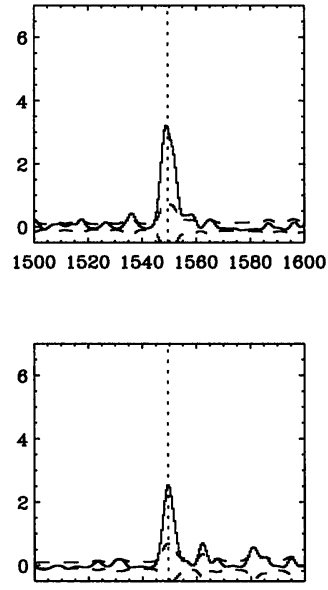

150015201540156015801600

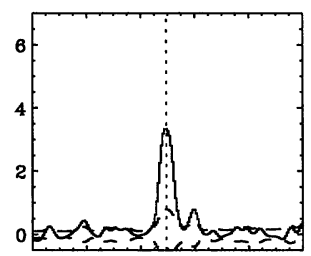

150015201540156015801600
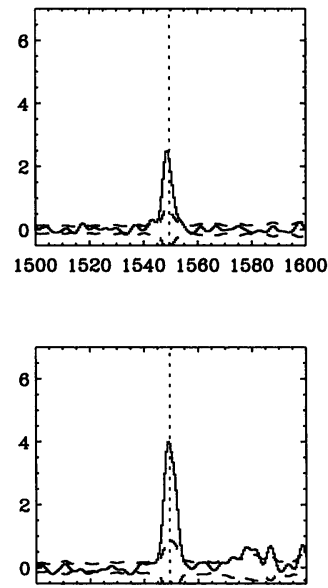

150015201540156015801800
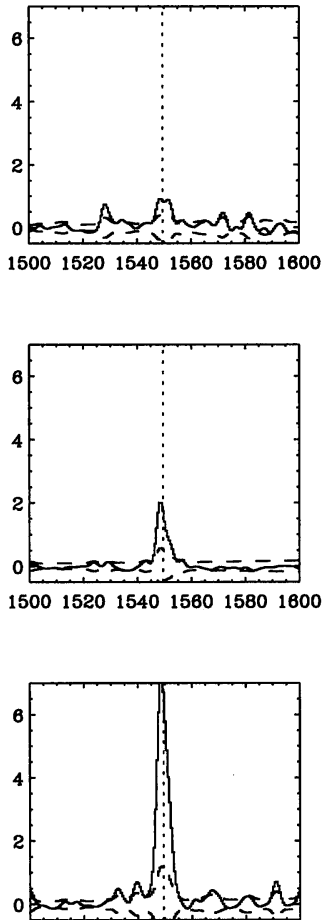

150015201540156015801600

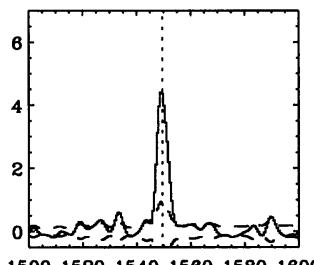

150015201540156015801600

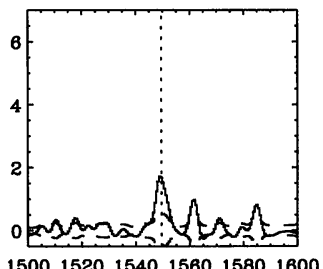

\section{Wavelength $(\AA)$}

Figure 1. Time series of C IV observations for the dM8e star VB 10. Each observation represents a 10 minute exposure. The dotted lines show the $1 \sigma$ error. Note the strong flare in exposure 7 . 
Additional figures and more extensive discussion of these results may be found in Hawley \& Johns-Krull (2003).

\section{Summary}

Our conclusion is that active, very low-mass, main-sequence stars (spectral types M7-M9) can maintain a quiescent chromosphere and transition region that is similar to those observed in active, earlier type $\mathrm{M}$ dwarfs. Flaring may still be ultimately responsible for the outer atmospheric heating in these stars, even during "quiescence"; the role of flares (particularly micro-flares) in coronal and transition region heating is still not understood for the Sun or for active stars (e.g. Kashyap et al. 2002; (Güdel et al. 2003). However, theories involving changes in atmospheric structure, dynamo heating and other mechanisms proposed to explain the lack of quiescent activity in very low mass stars and brown dwarfs must accommodate the persistent quiescent activity that we have observed at least through spectral type M9.

Acknowledgments. This research was made possible by grant HST-GO9090 from the Space Telescope Science Institute, operated by the Association of Universities for Research in Astronomy, Inc., for NASA.

\section{References}

Burgasser, A. J., Liebert, J., Kirkpatrick, J. D., \& Gizis, J. E. 2002, AJ, 123, 2744

Fleming, T.A., Giampapa, M.S., \& Schmitt, J.H.M.M. 2000, ApJ, 533, 372

Gizis, J. E., Monet, D. G., Reid, I. N., Kirkpatrick, J. D., Liebert, J., \& Williams, R. J. 2000, AJ, 120, 1085

Güdel, M., Audard, M., Kashyap, V. L., Drake, J. J., \& Guinan, E. F. 2003, ApJ, 582, 423

Hawley, S.L., Gizis, J.E., \& Reid, I.N. 1996, AJ, 112, 2799

Hawley, S.L. \& Johns-Krull 2003, ApJ, 588, L109

Johns-Krull, C.M., Valenti, J.A., \& Linsky, J.L. 2000, ApJ, 539, 815

Kashyap, V. L., Drake, J. J., Güdel, M., \& Audard, M. 2002, ApJ, 580, 1118

Liebert, J., Kirkpatrick, J.D., Reid, I.N., Fisher, M.D. 1999, ApJ, 519, 345

Linsky, J.L., Wood, B.E., Brown, A., Giampapa, M.S., \& Ambruster, C. 1995, ApJ, 455, 670

Martín, E. L. \& Bouy, H. 2002, New Astronomy, 7, 595

Mohanty, S., Basri, G., Shu, F., Allard, F., \& Chabrier, G. 2002, ApJ, 571, 469

Rutledge, R.E., Basri, G., Martín, E.L., Bildsten, L. 2000, ApJ, 538, L141 